\title{
Tailoring the Development Process According to the Context of the Project
}

\author{
N. du Preez ${ }^{1}$, D. Lutters ${ }^{2}$ and H. Nieberding ${ }^{1}$ \\ ${ }^{1}$ Department of Industrial Engineering, University of Stellenbosch, Stellenbosch, South Africa \\ ${ }^{2}$ Laboratory of Design, Production and Management, Faculty of Engineering Technology, \\ University of Twente, Enschede, The Netherlands
}

\begin{abstract}
As part of a research project on design methodology, the authors have developed a framework to facilitate the tailoring of the product development process according to the context of the project. In the first part of this paper, the reader is introduced to the framework. The second part presents two case studies conducted during the Management of Product Development course at the University of Twente. The case studies were intended to validate deployment of the framework to facilitate the selection and/or tailoring of the development process of a chosen product. This depends on the circumstances surrounding the product and the organisation that develops it.

The first case study analyses the development process proposed by students for a product they chose, without exposure to the framework. In contrast the second case study analyses the proposed development processes of a group of students after they were exposed to the framework. This paper presents the differences observed in the proposed development processes when the major variables that influence this process are highlighted.
\end{abstract}

\section{Keywords:}

Engineering design and development, development process

\section{INTRODUCTION}

\subsection{Models of the product development life cycle}

In order to improve the quality and efficiency of the development process, researchers in the field of design research have modelled the life cycle of the product development process since the 1960's. Within the engineering fraternity models range from the simple models such as the one by French [1] to very complex models, for instance, the model for systems engineering [2]. The model described in the guideline number 2221 of the Society of German Engineers (VDI) [3] is a typical example of average complexity. Generally these models are characterised by the following [4]

- prescriptive sub-division of the development process into phases each generating in specific intermediate results,

- sub-division of the main problem into sub-problems, each sub-problem is solved and the main solution is generated by integrating all sub-solutions,

- each sub-solution is generated by selecting the most promising one of multiple alternatives, which are based on the analysis of the functional requirements.

Although the proposed models for the development process among engineers were initially similar to those in the architectural domain, architects gradually seem to have favoured descriptive models such as the one by March [5]. These models are characterised by the following [4]:

- descriptive emphasis on the cognitive processes within the development process,

- a main problem is solved by focusing initially on the central problem, and addressing all peripheral problems accordingly [6], and
- solutions are generated early in the development process based on presuppositions.

The engineering practitioner is presented with a large number of diverse models and methods related to the process of developing products. How does one choose the development process for a given, specific project?

\section{FACTORS INFLUENCING THE DEVELOPMENT LIFE CYCLE}

The authors agree with researchers such as Stetter [7] and Maffin [8] that development life cycles have to be adapted to suit the boundary conditions (context) of the specific development problem. They therefore initiated a research project to determine how the models of and methods for product development generated by design science can be adapted to suit the context and constraints of development projects in the 'real world'. To this end the authors identified parameters, based on Maffin's [8] contextual framework, that influence the development life cycle. These are depicted in Figure 1 and discussed in the following sections.

\subsection{Variables related to the organisation}

Dumas and Whitfield [9] have indicated in their research that "industry, in its relationship with the design function, is not a uniform domain which can be addressed simply and directly; rather it is segmented with each type exhibiting a unique culture/practice profile. As such, it argues against a simplistic approach to the management of design within industry and suggests a greater need for tailor-made solutions". The word organisation in this context represents the environment in which the product is to be developed, including such issues as client and supplier involvement. 


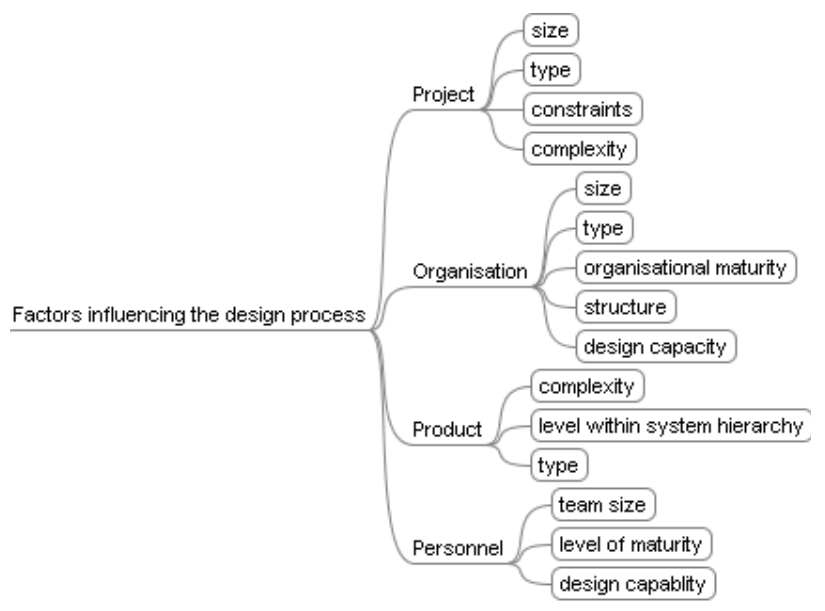

Figure 1: Parameters influencing the development nrocess

\section{Organisational size}

The influence of the size of the organisation on the development life cycle is similar to the effect that the size of the project team has on the development life cycle (see section 2.2). The interrelationship among variables related to size is discussed in section 2.5. In general, the larger the organisation (or project team) the greater the managerial control, and therefore the more formal and prescriptive the development process. Jones et al [10] quantified typical attributes of an organisation (project) according to its size.

As a variable describing the organisation, which will be performing (or at least managing) the development process, organisational size describes in general terms the amount of available resources and implicitly the manner in which the organisation's business in general and product development in particular is approached. This is reflected in Koeller's research [11] which indicates that large firms can afford to be innovative in industries which require substantial advertising and capital intensive investments. On the other hand small firms find it easier to be innovative in industries where an "entrepreneurial mode of behaviour" is favoured.

Radcliffe et al [12] have described the working environment of a typical small manufacturing business in Australia. Due to the limited availability of resources [13], they often employ no professional engineers, reducing their exposure to formal design methodologies and methods. Key personnel in the organisation usually "perform multiple roles" and "a diverse range of tasks without the option of delegation". This means that development projects have to be fitted in between the daily tasks required for the running of the business. As a consequence internal communication tends to be verbal, effective [14], yet informal with frequent in-the-corridor meetings", resulting in very little information being recorded, collated and reported in a formal structured manner. Therefore "much of the daily working knowledge of the enterprise resides in the memory of the staff ", "poorly supported by documentary evidence, and strongly centred in the moment", resulting in a "tendency to concentrate attention on short term rather than ultimately more important strategic issues".

Conversely, the availability of resources makes it possible to appoint personnel for a specific task, such as the development of a new product. Strategic issues to be resolved are addressed by the relevant departments without interruption of the current production crisis or customer complaint. Not only are resources available to record, collate and report information, it is imperative that this is done to ensure effective and efficient communication. The co-ordination and management of a large company cannot be achieved by in-the-corridor meetings. This structure results in a "resistance to change and organisational inflexibility that often characterise larger firms" [13]. Walsh and Roy [15] determined through a study that "the transition to formalised management structures occurred when a firm reached about 100 employees".

Author 3 (Nieberding) of this paper, has worked in South African companies of various sizes, and can confirm through experience that these descriptions are also typical for South African companies.

\section{Organisational structure}

Dumas et al [9] have indicated how the following two variables related to the internal structure of an organisation can influence the way that product development is implemented:

- the existence of the position of design manager, and

- whether the company operates in the service or manufacturing sector.

Companies employing a design manager, seem to assign greater importance to the design function within the company, operating the design department as a profit centre with a high level of accountability. In comparison companies without a design manager prefer central control of design projects with accountability diffused among finance, engineering and marketing.

In the manufacturing sector the design function is dominated by engineering, internal design teams are utilised and accountability is retained by the designers. In contrast the design process in service industry is dominated by marketing and is reliant on external consultants and design policy documentation.

Maffin [8] determined that the "degree of autonomy over the strategy-making process" is important: a department or team of a large organisation can be given authority to plan and execute a project autonomously from the rest of the company, or conversely, if the unit is forced to operate within the constraints of the larger organisation, generally a homogeneous (and therefore prescriptive and static) approach will be followed, that fits the requirements and philosophy of the larger organisation, rather than those of the smaller design team. Brown and Eisenhardt [16] call this autonomy "subtle control", where senior management communicates the vision for the new product and then provides "enough autonomy to be motivated and creative".

Organisational type

Purpose The first aspect is the organisation's main purpose [8]: is it trying to be a product leader, i.e. develop products which excel in performance, quality and reliability, or an effective manufacturer, i.e. concentrate on effective manufacturing and cost minimisation? The first can be seen as a product developer with a manufacturing facility, while the second is a manufacturing plant with a design office to handle minor changes to improve efficiency and cost. Typically the former will concentrate on the initial part of the product life cycle by generating the product definition and documentation, producing prototypes and assisting in the generation of pre-production models. The latter typically obtains the product definition from the former and produces the product in volume (the second half of the product life cycle). 
Cooper [17] describes how the development process is dominated by marketing activities if the new product is driven by the demands and requirements of the market (market orientation). Conversely, the development process is dominated by technical and production issues if the development is enabled by technological advances (design domination and/or prototype domination).

Production size and inventory philosophy These aspects of the type of organisation are clearly related to the organisations main purpose as discussed above. Production size can either be unit, batch or mass production for discrete products. The inventory philosophy can be one of four options: [make-to-stock, assemble-toorder, make-to-order or engineer-to-order]. Generally products engineered-to-order are produced in unit or batch production, while make-to-stock products lend themselves to mass production. As a parameter of the organisation, production size influences the development life cycle by defining the norm for the company. The effect of production size on the development life cycle as product related parameter is discussed in section 2.3. The point being that a company accustomed to producing a specific product type, for instance engineer-to-order, will have to change the development methodology if it should decide to develop a made-for-stock product.

Selling products The organisation's mode of selling products is related to the production size and stock philosophy:

- selling standard products through catalogues,

- selling customised products and competing through tenders

- suppliers operating with the supply chains of a limited number of customers, establishing long term sales agreements.

Products produced in mass production lend themselves to long term sales agreements, since this agreement lowers the risk for investing in the establishment of capital intensive mass production facilities. Engineered-to-order products are often customised products sold through tenders, due to the fact each customer has different, specific requirements. As a third variant, make-to-stock, assemble-to-order and make-to-order can be standard products which are sold through catalogues, where the customer can choose a specific configuration made up of a combination of standard components.

Organisational maturity

Organisational maturity relates to the management of processes within the organisation as defined by Jones et al [10].

Project Management The process of managing all aspects of the project, i.e. technical integrity and quality, timescales, financial expenditure.

Documentation The process of capturing information during the design process for internal communication as well as support to the customer.

Computer \& Tool Support The support of the use of computers and design tools during the development process.

Tool use learning The process followed to acquire the skills to use new tools effectively and efficiently.

Selection of tools The process of deciding which tools are applicable to the specific development process.

These aspects can be implemented during the development process in a number of ways:

Level one refers to processes which is managed and executed in an ad hoc fashion. At the second level each team member may implement some of these aspects to some degree, but no overall philosophy is applied. On the third level a common philosophy towards these aspects is accepted and implemented by the whole project team, although this may vary from project to project and from team to team. For the fourth level organisation wide consensus is obtained with regard to the philosophy of managing and executing development projects. The last level indicates that the development process is evaluated on a regular basis to determine how it can be improved.

It is reasonable to assume that an organisation's level of maturity with regard to the management of aspects described above will also be reflected in the planning, management and execution of the development process. A company at maturity level one, may have a very unstructured, uncoordinated approach to the design of their products. In comparison an organisation situated generally at level four or five, will find it easier to have a uniform approach to product development and evaluate this approach on a case-by-case basis to maximise the performance of the development process.

\section{Organisational design capacity}

Should the organisation's design resources not be sufficient to develop a specific product, the organisation can either employ design staff or sub-contract a portion of the design. For the former provision has to be made in the development process for training and/or mentoring of the new employees, while for the latter, the development project should be structured in such a way that the subcontracted portion consists of a definable sub-system or module to facilitate the interface of the sub-contracted work with the rest. Not only is the structure of the development process different, the type of activities and the required capabilities also differ. The issue of in-house capacity is therefore often linked to in-house capability (section 2.4): if the company does not have the necessary capability to execute a portion of a project, it subcontracts it.

\subsection{Variables related to the project}

Shenhar and Wideman [18] describe a project as a process, "a journey through time", where the "boundaries or limitations imposed on the journey" are "expressed in terms of scope, quality, time and cost". In the case of a product development project, the outcome of that journey is the definition of a product and its production process.

\section{Project size}

Project size relates to the number of resources required for a specific project [10]. It should be intuitively obvious that project size is related to project complexity, since it will take a larger project team to develop a complex system in the same time frame.

Similar to the effect of organisational size, increasing project size requires increasing managerial control and therefore a more formal structure of the development life cycle, increasing the amount of project documentation due to the increased coordination and communication efforts. Therefore large projects may be forced to follow all the phases of the development process in a structured manner, not because of the work content, but rather as a part of the co-ordination and communication effort. Due to the informal and efficient communication channels in small organisations (teams), the development process can often be significantly streamlined by reducing (or even removing) some of the phases. This is often referred to as the "skunk works approach".

\section{Project complexity}

Project complexity has the same effect as project size on the development life cycle, due to the fact that large 
projects are usually inherently complex. However, even the development of simple projects can have complex projects. For example, projects requiring the involvement of external organisations, such as legal experts or approval authorities. According to Günther [19] complexity has the following attributes:

Complicacy A problem with a large number of variables is more complex,

Dynamics Time dependent problems, requiring intervention at the correct time,

Opacity Only a portion of the relationships among project variables are visible at any time,

Interdependency Project variables are interrelated so that unintentional side-effects are created by adjusting project variables with the aim of creating a beneficial effect, and

Multiple objectives (German: Polytelie) Striving for the achievement of multiple, conflicting goals simultaneously.

Therefore complex projects may require a very formal and structured development process in order to integrate the various aspects of the project.

Project type

Ullman [20] defines the project type as follows:

- original design is a new development, which is not based on a previous product or idea;

- parametric design, where parameters are adjusted to suit requirements;

- configuration design, which requires mainly the packaging of existing modules or components;

- selection design, where the correct standard component to suit the requirements is selected from a catalogue and

- redesign, which requires modifications to an existing product to meet new requirements.

Selection design typically requires the least amount of engineering effort, is technically quite simple, and this should be evident in the extreme simplicity of the development life cycle. Parametric design, configuration design and redesign project are typically more complex and technically challenging, representing the majority of projects in practice. These projects focus on the changes needed to meet the new requirements. Obviously the original design problem requires the complete development life cycle from concept phase to detail phase, as described in design literature, such as the VDI guideline [3].

\section{Project constraints}

Project constraints that affect the development life cycle are typically time or project duration, cost and technical risk. These are conflicting variables. To minimise the project duration, additional resources (personnel, subcontractors) are required, as well as highly efficient communication and strong project management. The minimisation of cost is highly dependent on the type, complexity and risk of the product to be developed. Cost could be reduced by favouring computer simulation rather than the construction of prototypes; or modules or subsystems could be purchased rather than developing them from scratch. Technical risk is strongly linked to the project attribute, level of novelty, discussed below.

Project novelty

The word "novelty" refers to the new aspects of the project, the unknown. Novelty does not necessarily mean that it has never been done before; it could merely mean that it has not been done by this specific company or project team before. Frost [21] defines the level of "novelty" or innovation required as follows:

- If the designed entity is already known in stereotype form, and parameter values only (not configuration) need to be determined, then a very low degree of innovation is required.

- If no viable pre-existing stereotype is available, so that configuration must be fully determined, then the design is revolutionary and a high level of innovation is required.

Design is described as a heuristic process, i.e. the exact process to follow and the expected results are unclear at the start of the process, and are clarified during the project by learning about the subject matter. This is especially true for projects with a high novelty content. High levels of novelty therefore require adjustments to the development life cycle to accommodate the obtaining of information, learning, experimentation [18] [16], feedback loops, even set-backs and failure.

\subsection{Variables related to the product}

\section{Product type}

Product type defines the intended production quantity for the product, either unit, batch production or mass production. Whereas production size, discussed in section 2.1 , is a characteristic of the company, reflected in its business philosophy and processes, the product variable addresses the fact that a product's intended production quantity has an effect on the design of the product and is therefore a characteristic of the product rather than the entity that produces it.

Hykin and Laming [22] found that the development of mass produced products require emphasis on the first phases of the process to ensure the design of "a nearly right solution for the final design phase and production", requiring a "formal, rigidly defined system of control with a well-developed command hierarchy". For mass-produced products concurrent engineering can contribute greatly to the reduction in development times, since the production process is just as important as the product itself. In contrast the development of one-off products is "less ordered and constrained" with an organisational structure which is "less formal and more adaptable", attempting to generate as much flexibility as possible in the early phases to delay detail decisions until the final phases of the project.

Frost [21] indicates that the intended production quantity introduces "implications for the optimal trade-off between development time and costs, on the one hand, and production or building costs, on the other hand".

Product complexity

Product complexity can be defined in terms of complicacy, opacity, interdependency and multiple objectives, similar to project complexity (see 2.2). The development of low complexity products generally shows a short concept phase, no embodiment phase and moves directly into the detail phase [8]. High complexity products, however, require a definite concept phase and a distinct embodiment phase to bridge the gap between the overall design concept and the detail requirements.

The findings of Shenhar and Wideman [18] indicate that increasing complexity increases "the need for higher and more formal project management".

Some prescriptive development methodologies (see [3]] for example) maintain that design problems can be dissected into sub-problems which are addressed more or less in isolation, due to an assumption that design problems can be structured in a hierarchical manner. The 
resulting sub-solutions can then be re-assembled to generate the total solution for the original design problem. Although this may be true for simple design problems, it is the authors' experience that this view does not hold for complex products such as motor vehicles, ships and aeroplanes.

However, natural processes and complex design problems result in a lattice structure, since most (or even all) aspects of the design problem are interrelated to most other aspects. By dissecting the problem in to subproblems, some of these interrelationships are severed, resulting in optimised sub-solutions, rather than an optimised solution for the complete problem. The only way to address these type of complex design problems is in the manner described by the "descriptive" models of the development process, where the central (most important) aspect of the design problem is addressed first, incorporating the rest of the design problems in the process. The total solution therefore evolves and changes to provide acceptable solutions for the minor aspects of the design problem.

The product complexity is often related to its level in the system hierarchy, and this aspect is discussed below.

\section{Product level of hierarchy}

Günther [19] provides a typical level of hierarchy: plant, machine, sub-system, and component. The effect of the level of hierarchy on the development life cycle is similar to the effect of product complexity discussed in the section above, since the complexity increases with increasing level of hierarchy.

On the other hand, Frost [21] argues that products on high levels of hierarchy, such as plants, vehicles and ships, although complex, can typically be conceptually decomposed into subsystems. "The more this can be done, the easier will be the design, the less holistic the approach need be and the more people will be able to work effectively and simultaneously on the design once physical and performance interfaces between systematically adjacent subsystems have been defined."

\subsection{Variables related to the personnel}

\section{Team size}

Team size is strongly related and has the same influence on the development life cycle as the size of organisation and project size discussed in sections 2.1 and 2.2 respectively. The fact that these three variables are not necessarily identical is discussed in more detail in section 2.5. Organisational size influences the development project by transferring at least some of the management philosophy from the organisation onto the project. While project size defines the resources that the project requires to complete it in a given time-scale, team size defines the resources actually allocated to the project. In the authors' experience the projects size usually exceeds the team size.

\section{Level of maturity}

According to Ehrlenspiel and Dylla [6] mature designers

- are more precise and spend more time on analysis and formulation of requirements.

- increase the scope during search for solutions reformulation and summary (see also [23].

- approach problems strategically from important to less important sub-problems.

- generate variants especially in important problem areas.

- are more accurate during and spend more time on the analysis of solutions.
- have better spatial imagination.

- apply adequate and meaningful strategies for steering the design process based on thorough analysis of demands and solution properties.

These attributes influence the required detail and structure in the planning, management and execution of the development life cycle. It is the author's experience that mature, experienced personnel will use their experience to determine

- which parts of the design problem constitute the core of the design problem, requiring the main attention, and

- the level of "structuredness" required for the design problem dependent of the level of complexity and novelty (see 2.5).

To compensate for their lack of experience less mature designers will require a more structured approach. Any project may seem complex and novel, due to the fact that they have very little experience to judge complexity. Thus the possibility exists that sub-problems and the core problem are addressed with the same approach, resulting in inefficiencies and longer development times.

The paragraphs above have mainly focused on the maturity and experience of technical team involved in the development process. However the success of product development is also influenced by the maturity, experience and involvement of senior management [24] "by granting authority to the project manager"and by "defining the (development) problem strategically".

\section{Design capability}

The variable "design capability" is related to maturity above and is defined by Günther [14] as a combination of epistemic and heuristic competence. The former is defined as technical knowledge, composed of theoretical knowledge (know what) and procedural knowledge (know how). The latter is defined as the capability to develop and implement strategies in new and complex situations. One would therefore expect that "design capability" increases with increasing "maturity".

Cantamessa [25] describes the capabilities of an organisation "as a collection of decision rules and routines tied together by the firm's internal language and communication code. Part of this knowledge may be codifiable and transmissible, whereas another part may be tacit and embedded in individuals; in the same way part of the knowledge may be formal, based on theoretical understanding, whereas another part of the knowledge may be procedural and based on experience".

\subsection{Framework for the contextualisation of the development life cycle}

To clarify the variables discussed in the previous sections, and their interrelationships, the author has arranged them in the form of a framework, shown in figure 1 . The framework is aimed at making the engineer, who has to plan, manage and implement a development project in practice, aware of the main context variables that influence the development life cycle.

Although these main context variables have been discussed individually in the preceding sections, some of the variables are interrelated, and it is important to consider these relationships in a holistic manner.

\section{Variables Related to Size}

Organisational size just indicates how many people work for the organisation which is executing the project, while project size determines how much manpower is required to execute the specific project, and the team size 
describes the number of people actually working on this specific project. In all three cases the required management and control increases as these sizes increase, and therefore the effect on the development methodology is clear as long as the variables are in harmony. However, when a large organisation selects a small team to fast-track a development project, the tendency might be to enforce the philosophy associated with a large organisation on to the small team, increasing the overhead burden unnecessarily.

Similarly, when a small company, accustomed to managing small teams and small projects, has obtained an order for a project, which necessitates a large team, work is sub-contracted, suppliers are drawn into the development process, new people are employed, and the successful execution of this project requires much more managerial control and formal communication channels than the usual small project. In both these examples the project requirements in terms of size were matched with the team size selected to execute the project. It is sometimes (even often) the case that the required manpower is not available (design capacity) and employing additional people is not feasible due to the short timescales involved in the project. In this case the need for control and formal communication has to be balanced with the fact that the team members are already overburdened due to the lack of resources. In this case effective teamwork is of utmost importance. Easy access among the team members through working in the same location and frequent verbal communication will contribute to the success of the project.

\section{Variables Related to Type}

Another set of related context variables are product type and organisation type. As with the examples above, careful balancing of conflicting aspects of the development methodology is required when these variables do not match. For instance, a product, to be manufactured in small batches, is designed by or for an organisation, which usually mass produces products. If the development is done by a consultant, the differences in production philosophy and the resulting consequences should be addressed during the development process to prepare the manufacturing organisation properly. If the development is performed in-house, besides addressing the issues mentioned above, the organisation will be unaccustomed to the suitable development process.

\section{Variables Related to Complexity}

Project complexity is determined to a large degree by the product complexity. The development of large complex products such as aeroplanes, mining operations or international airports require, by their very nature, complex development processes. The balance to be found here is to make the development process as simple as possible, but as complex as necessary.

The relationship between complexity of project and product on the one hand and the "structuredness" of the development process on the other is not linear. For "simple" products the structured approach of dissection and re-assembly of the development problem (see section 2.3) is easily applied due to the fact that the interrelationships between requirements of the product are relatively few and straight forward. The interrelationships between requirements of products with medium complexity are too complex to handle easily via a structured development process (e.g. [3]). In the author's experience these type of products are developed more efficiently by employing the approach advocated by the "descriptive" models of the development process: focusing the design effort intuitively onto the central design problems first, making the peripheral problems subservient to the solutions of the central problems, without loosing sight of the integration of the whole. In his career author 3 has come across projects where the dissection and re-assembly process of a structured development process was implemented without careful consideration for the interrelations between the subproblems, invariably resulting in a unsatisfactory product. For highly complex projects, the amount of integration required is too much for a single person to manage. There is therefore no choice but to subdivide the whole problem into "palatable" chunks by means of the "standard" structured approach. Obviously care will still be required in the assembly or integration of all the subsolutions into the final solution to the development problem.

Rzevski [26] describes the threshold between problems of medium and high complexity as described above as "perceivability": "the ability to hold all the important aspects of a problem and all their interrelationships in mind at one and the same time".

\section{Variables Related to Maturity}

Organisational and personnel maturity are complimentary. Mature experienced design personnel is required to obtain an organisation with a mature development process. Organisational size also plays a role: the process of a small company may seem immature due the lack of necessity for structure and formality, even though all aspects of the development process are managed diligently and all risks are addressed with care. Conversely, the development in a large company may seem mature, where in fact it is rather cumbersome and inefficient.

\section{Variables Related to Capacity and Capability}

Design capacity is expressed in man-hours available to execute projects and is therefore seen as an attribute of the organisation rather than the personnel. Design capability is defined as the skill and competences that each individual makes available for the organisation to utilise, and therefore considered an attribute of the personnel rather than the organisation.

\section{THE USE OF THE FRAMEWORK IN CASE STUDIES}

The first case study to evaluate the framework of parameters (figure 1) was conducted as follows. Students taking the Management of Product Development course of the Deptartment of Industrial Design at the University of Twente are asked to select a product and describe its development process. In 2006 they were given this task without exposure to the framework, while in 2007 the students were presented with the information summarised in section 2 above.

Generally, the quality of the articles of 2007 had improved, focussing more on the realities surrounding the product and the organisation that produces it, rather than fairly vague concepts such as designer freedom and employee satisfaction.

In 20065 articles of 23 (i.e. 22\%) did not consider these factors at all in their proposed development processes, while only 2 articles (or $8 \%$ ) mentioned whether the development was to be performed in-house or by external sub-contractors. In comparison all students in 2007 made a decision, where the design capability would reside. The percentage of students who decided to adapt a standard process to suit the context of the project increased from $26 \%$ in 2006 to $47 \%$ in 2007 . 


\section{ENVISAGED SITUATION}

Elaborating on the following concepts, it is conceivable to develop a repository of all available frameworks (generic models):

- a configurable development life cycle,

- the reuse of objects within previously constructed roadmaps, and

- the availability of a collaborative design environment that will support object oriented configuration and manipulation of roadmaps.

These generic models provide the objects to build specific roadmaps (partial models) for development of specific artefacts embedded in a specific context. Implementation of such a partial model to develop a specific product would create a uniquely identified and instantiated roadmap in the collaborative design environment. Generic models, partial models and previously executed roadmaps would be available for reuse in configuring roadmaps for future projects.

\section{CONCLUDING REMARKS}

Research into engineering practice has indicated that each development project requires a development life cycle, tailored to suit the context of the specific project. The authors of this article have proposed a framework of contextual variables, which influence the development life cycle. Each variable and the way it influences the development life cycle were discussed briefly.

The concepts summarised in the framework were presented to students of the University of Twente, and the results indicate that the use of the framework increased the awareness of the students with regards to the context surrounding a development project that influences the development process.

The fact that development life cycles are time dependent, require the development and modification of life cycles "on the fly". Coupled with the tendency to develop products by diverse (often distributed) teams, the need for a collaborative design environment was highlighted.

The reuse of theoretical frameworks and models within the extensive knowledgebase of design science, as well as the past experiences residing within each development environment, would necessarily increase the quality of the development life cycle for a new product. Therefore, the need was indicated for the establishment of a repository of existing frameworks, models, life cycles and roadmaps. Combined with a design environment to manipulate this existing information, a specific instantiation of a life cycle for the development of a specific product within the context of a specific environment can be constructed.

It is the authors' opinion that this collaborative design environment and the associated repository, which provide the ability to configure custom-made, dynamic development life cycles, will

- facilitate the learning process within an organisation from previous mistakes and successes,

- greatly expedite the technology transfer from design science to industry, and

- increase the acceptance and use of frameworks, methods and models of design science in industry.

\section{ACKNOWLEDGEMENTS}

The authors would like to thankfully acknowledge the ongoing support and assistance received from South African companies: Indutech (Pty) Ltd, www.indutech.co.za, Mechanology (Pty) Ltd, www.mdbsa.com, BAE Landsystem OMC,

www.baesystemsomc.co.za, as well as the support of the Global Competitiveness Centre in Engineering. (www.gcc.co.za).

\section{REFERENCES}

1 French, M. J., Engineering Design - The Conceptual Stage, 1971

2 Blanchard, B. S. and Fabrycky, W. J., Systems Engineering and Analysis, 1998

3 VDI 2221, Systematic Approach to the Development and Design of Technical Systems and Products, 1993

4 Cross, N. and Roozenburg, N., Modelling the Design Process in Engineering and in Architechture, Journal of Engineering Design,3,4, 1992

5 March, L. J., Systematic method for designers, 1984

6 Ehrlenspiel, K. and Dylla, N., Experimental Investigation of Designers' Thinking Methods and Design Procedures,Journal of Engineering Design,4,3, 1993

7 Stetter, R., Method Implementation in Integrated Product Development, 2000

8 Maffin, D. J. B., Engineering Design Models: Context, Theory and Practice,Journal of Engineering Design,9,4, 1998

9 Dumas, A. and Whitfield, A., Why Design Is Difficult to Manage: a Survey of Attitudes and Practices in British Industry,European Management Journal,7,1, 1989

10 Jones, D. A. and York, D. M. and Nallon, J. F. and Simpson, J., Factors Influencing Requirement Management Toolset Selection,2, 1995

11 Koeller, C. T., Innovation, Market Structure and Firm Size: a Simultaneous Equations Model,Managerial and Decision Economics,16, 1995

12 Radcliff, D. F. and Harrison, P., Transforming Design Practice in a Small Manufacturing Enterprise, 1994

13 Roy, R. and Potter, S., Managing Design Projects in Small and Medium-Sized Firms, Technology Analysis and Strategic Management,2,3, 1990

14 Rothwell, R. and Zegveld, W., Innovation and the Small and Medium Sized Firm, 1982

15 Walsh, V. and Roy, R., Plastics Products: Good Design, Innovation and Business Success, 1983

16 Brown, S. L. and M., Eisenhardt. K., Product Development: Past Research, Present Findings and Future Directions,Academy of Management Review,20,2, 1985

17 Cooper, R. G., New Product Process: An Empirical Based Classification Scheme,R\&D Management,13, 1983

18 Shenhar, A. J. and Wideman, R. M., Optimizing Success By Matching Management Style to Project Type, 2000

19 Günther, J., Individuelle Einfl।"usse Auf Den Konstruktionsproze $\beta, 1998$

20 Ullman, D. G., Mechanical Design Process, The, 2003 21 Frost, R. B., A Suggested Taxonomy For Engineering Design Problems, Journal of Engineering Design,5,4, 1994

22 Hykin, D. H. W. and Laming, L. C., Design Case Histories: Report of a Field Study of Design in the United Kingdom Engineering Industry,Porceedings of the Institution of Mechanical Engineers, 189,23, 1975

23 Christiaans, $H$. and Dorst, K. and Cross, N., Levels of Competence in Product Designing, 1993 
24 Song, X. M. and Souder, Wm. E. and Dyer, B., A

Causal Model of the Impact of Skills, Synergy and Design Sensitivity On New Product Performance,Journal of Prod Innovation Management, 14, 1997

25 Cantamessa, M., Design Best Practices At Work: an Emperical Research Upon the Effectiveness of Desgin Support Tools, 1997

26 Rzevski, G., On the Design of a Design

Methodology,Design: Science: Method, 1981 